\title{
Identification of geodynamic movements based on the results of geodetic monitoring measurements
}

\author{
Andrey Panzhin ${ }^{1 *}$ \\ ${ }^{1}$ Institute of Mining, Ural Branch of the Russian Academy of Sciences, 620075, Russia, \\ Yekaterinburg, Mamina-Sibiryaka str., 58
}

\begin{abstract}
The methodology and results of an experimental study of modern geodynamic movements using the data from geodetic deformation monitoring are presented. The related issues of modeling natural, anthropogenic, and ecological systems are considered. A technique for visualizing geodynamic movements based on the results of cyclic geodetic measurements, which allows to reasonably identify active geological structures, blocks, tectonic faults, is presented. It is shown that as a key source of information on geodynamic movements over large spatiotemporal intervals, it is advisable to use not the absolute values of the vectors of displacement of observation points, but their velocities, normalized to the annual cycle. It is indicated that an important characteristic of the vector field of geodynamic movements is divergence, which characterizes the degree of convergence or divergence of the vector flow.
\end{abstract}

\section{Introduction}

The issue of the possibility of studying modern geodynamic movements using deformation monitoring data, carried out both in the mode of discrete observations at points of the geodetic network (GGS), and using data from permanent stations (GNSS), has been repeatedly considered in connection with the study of modern geodynamics of the Ural region, caused by both natural and anthropogenic factors $[1,2]$.

Identification of cyclic spatial coordinates of observation points and their changes in time is carried out, depending on the size of the networks, using Precise Point Positioning (PPP) methods [3] for large networks, and classical Double Difference (DD) methods for local networks. Also, PPP and DD methods can be used in combination, with the DD methods calculating and adjusting the geodetic network, and the PPP methods - linking local and regional geodetic surveys to the international IGS network.

An important aspect in the study and visualization of modern geodynamic movements is the modeling of natural, anthropogenic and ecological systems, enabling to reveal many properties and characteristics necessary for their analysis, including the hidden regularities of their spatial-temporal distribution. Meantime, much depends on the research adequate to

\footnotetext{
* Corresponding author: panzhin@igduran.ru
} 
the object and its components: identification and visualization of both quantitative and qualitative spatio-temporal information [4].

Also, visualization of geodynamic movements based on the results of cyclic geodetic measurements makes it possible to more reasonably identify active geological structures, blocks, tectonic faults, which is necessary to predict the locations of possible seismic events and take preventive measures to ensure the safety of people, industrial facilities, etc.

\section{Research Methods}

The studies of modern geodynamic movements in this paper were carried out at the Voronezh crystalline massif (VCM) using deformation monitoring data obtained by permanent GNSS stations in the region (Fig. 1). In order to be able to trace and evaluate the probable connection of modern geodynamic movements with the tectonic structure of the region, Fig. 1 combines the placement of GNSS points with the main faults in the region, marked by the geological materials.

Also, the epicenter of a seismic event attributed to natural earthquakes was recorded in the study area. The magnitude of the earthquake that occurred on March 31, 2000 was 3.9, and the depth of the seismic focus was 10 kilometers. In terms of the consequences, earthquakes of such magnitude do not pose a serious danger, but they, being a dynamic realization of the previous deformation processes, to a certain extent characterize the modern geodynamic activity of the region.

In total, in the study area of $200 \times 300 \mathrm{~km}, 18$ GNSS stations are located, which accumulate data, enabling them to perform high-precision geodetic reference to the global IGS network in the mode of cyclic monitoring measurements.

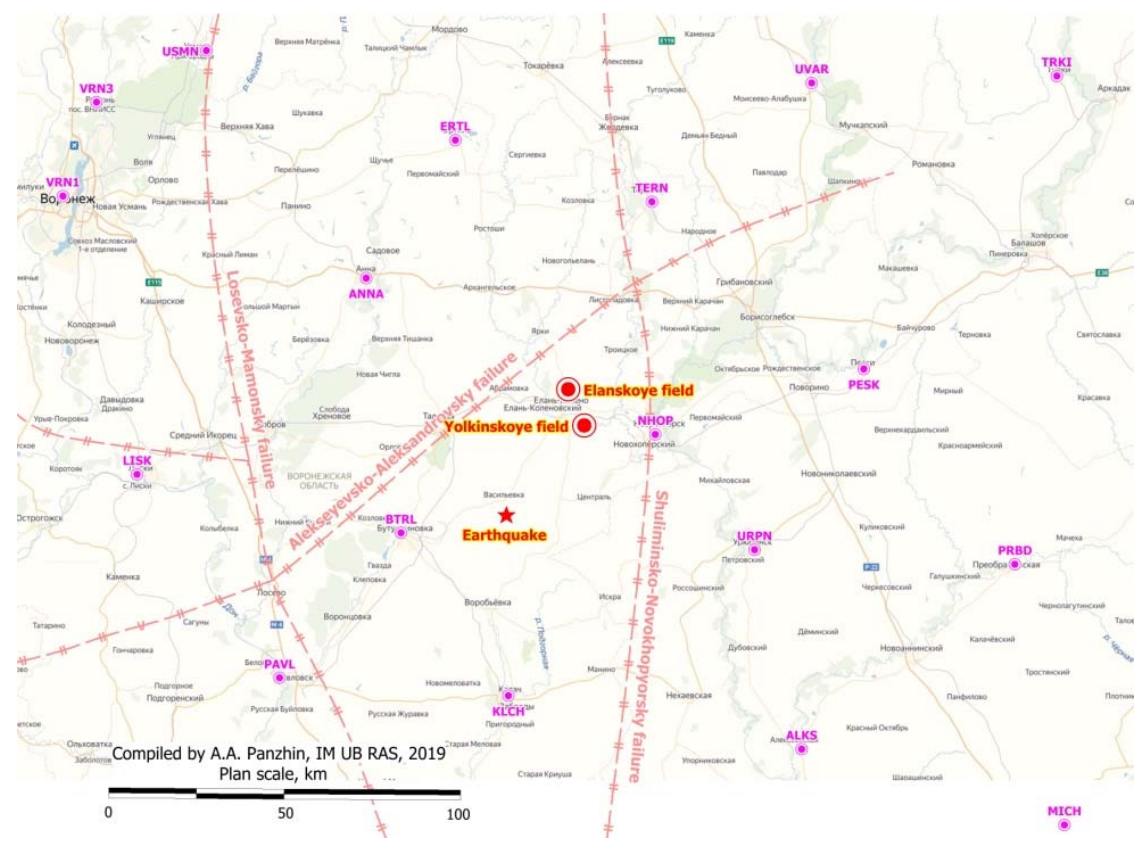

Fig. 1. Situational map of the location of GNSS stations, which were used to find modern geodynamic movements

In [5], it is shown that, as a key source of information on geodynamic movements based on the results of monitoring measurements carried out over large spacio-temporal intervals, it is advisable to use not the absolute values of the displacement vectors of GNSS points 
and benchmarks of observation stations, but their velocities normalized to the annual cycle. Normalizing the velocities to the annual cycle is necessary when carrying out monitoring using cyclic measurements irregular in time to bring the results to a common spaciotemporal basis.

\section{Results}

The annual velocities of the points trend movement vectors were found on the basis of an analysis of changes in their spatial increments of coordinates $\Delta \mathrm{X}, \Delta \mathrm{Y}, \Delta \mathrm{Z}$, which occurred in the intervals between repeated measurement cycles. The directions and values of the vectors are rather uniform, which reflects the general predominantly eastern trend direction of the lithospheric plate, including the VCM. As can be seen from Fig. 2, in the studied area, against the background of the trend component, a heterogeneity of the velocities of geodynamic movements is also noted.

As a key source of information on geodynamic movements based on the results of monitoring measurements carried out over large spatio-temporal intervals, it is advisable to use not the absolute values of the displacement vectors of GNSS points and reference points of observing stations, but their velocities reduced to the annual cycle. This is necessary when conducting monitoring using irregular time-based cyclic measurements to bring the results to a common spatio-temporal basis.

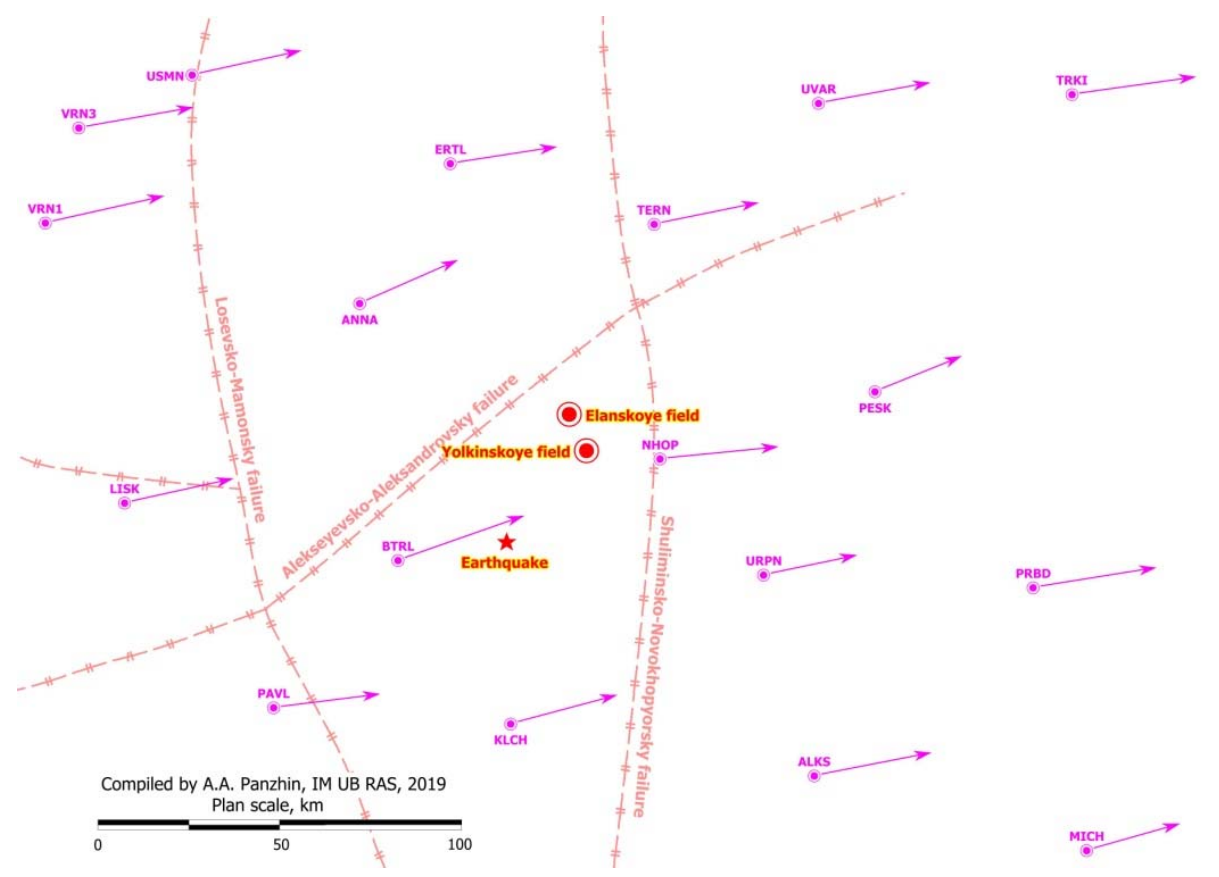

Fig. 2. Vectors of trend horizontal modern geodynamic movements

Also, an analysis of the velocities of geodynamic movements, and not of their absolute values, is advisable to be applied in the study of the displacement process in underground and open-pit mining of mineral deposits, since in the conditions of an operating mining enterprise there is a problem of loss of reference points of observation stations. This leads to a partial rotation of benchmarks due to the restoration of destroyed ones and installation of new ones on geodetic structures. At the same time, a comparison of the absolute values of displacements and deformations obtained in different periods of time will be incorrect 
when using traditional algorithms for assessing and analyzing the deformation state of the rock mass.

As a result, for each series of cyclic measurements, changes in the spatial increments of coordinates $\Delta \mathrm{X}, \Delta \mathrm{Y}, \Delta \mathrm{Z}$ of points are found, which, for the case of VCM, have a predominantly eastern direction due to the trend component. In this case, the vector field of deformations is visualized as almost uniform, which does not allow qualitatively identifying spatio-temporal information on geodynamic movements. For the numerical filtering of the trend component, the method of least squares was used, with the imposition of the condition of the mathematical minimum of the squares of displacement vectors along each axis of coordinates $\mathrm{X}, \mathrm{Y}, \mathrm{Z}$.

$$
\sum \delta \mathrm{x} \delta \mathrm{x} \rightarrow \min ; \sum \delta \mathrm{y} \delta \mathrm{y} \rightarrow \min ; \sum \delta \mathrm{z} \delta \mathrm{z} \rightarrow \min .
$$

As a result, the field of the vectors of geodynamic movements of the investigated VCM territory, in which the eddy displacements are visually identified, is found and graphically constructed, freed from the trend component (Fig. 3). The importance of visualizing eddy (rotational) geodynamic movements is explained by the fact that eddy boundaries are formed along the boundaries of large moving structural faults, which allows them to be identified as active tectonic structures of the region $[6,7]$.

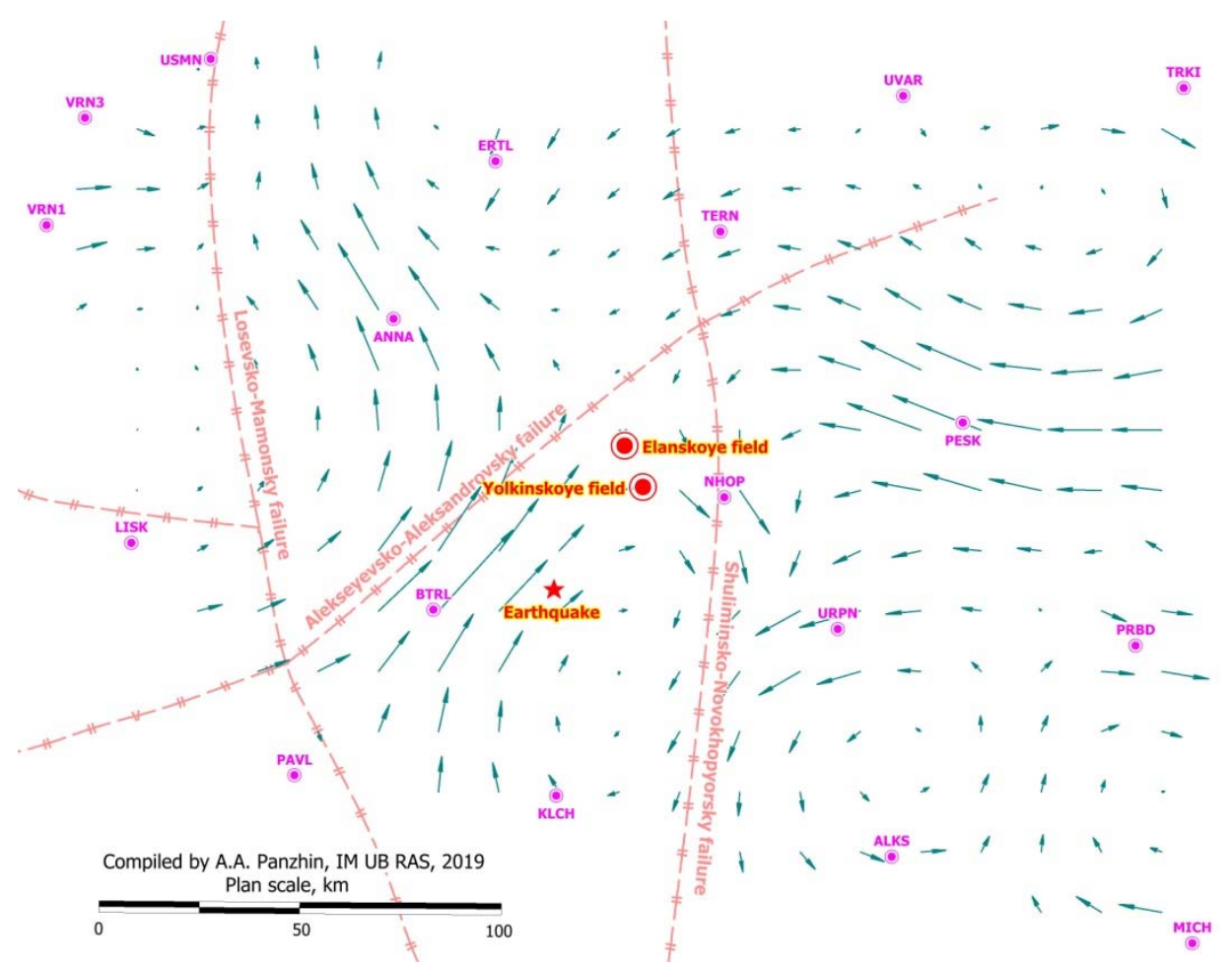

Fig. 3. Eddy motions in the study area

The geodynamic movements obtained as a result of measurements, using the mathematical apparatus of the continuum mechanics, are also transformed into a vector and tensor representation of the deformation field with the selection of the main components of the deformation tensor (Fig. 4). This makes it possible to find and identify the zones with the maximum values of the main deformations, both tension and compression, as well as the zones with increased values of shear strains, leading to the destruction of infrastructure facilities. 
Another important characteristic of the vector field of geodynamic movements is divergence, which characterizes the degree of convergence or divergence of the vector flow. Knowing the distribution patterns of its characteristics allows one to identify the sources of formation and flow of deformation processes, and their spatial position. The mathematical apparatus [8,9] has been developed and is being algorithmically implemented, enabling to find the divergence based on the results of the initial data presented both in the form of a uniform kriging model and in the form of data presented at the vertices of the unit elements in the Delaunay triangulation.

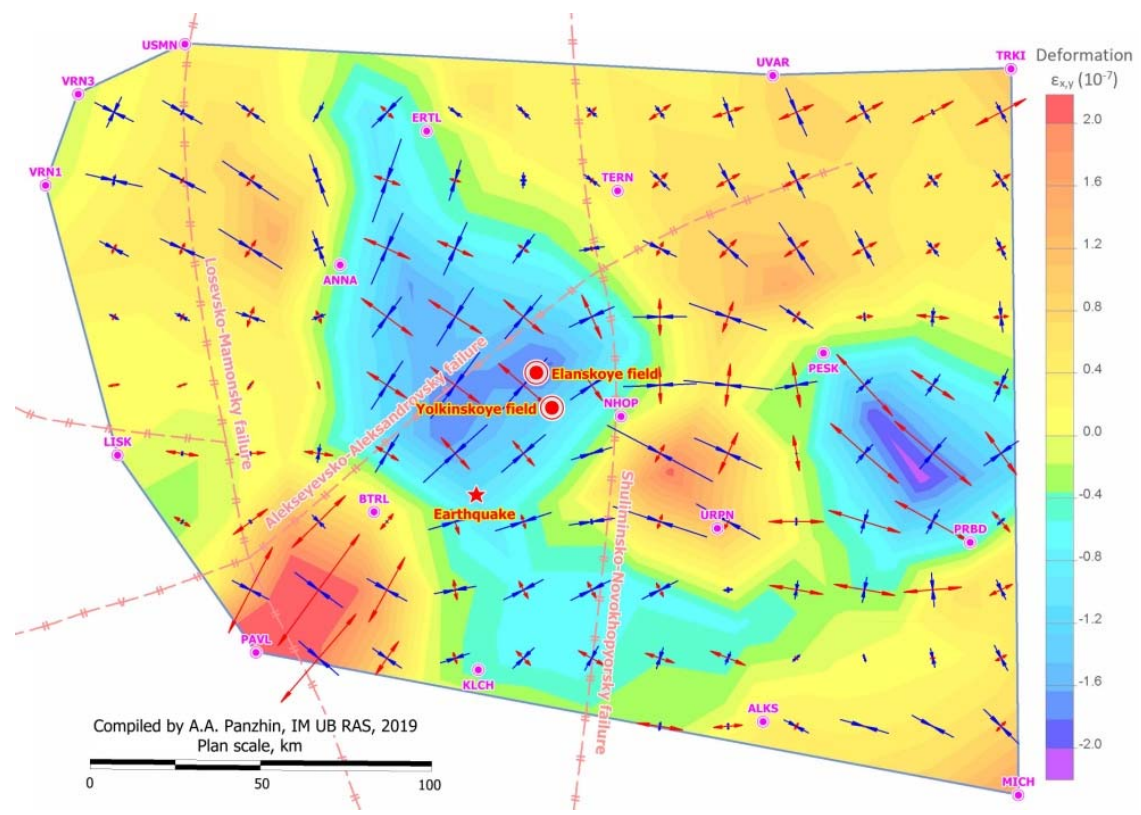

Fig. 4. Eddy motions in the study area

\section{Conclusion}

Thus, the paper outlines the main provisions of the methodology for monitoring and visualizing modern geodynamic movements in the form of a vector field based on the results of cyclic geodetic measurements. Based on the experimental data obtained at VCM using the results of deformation monitoring by permanently operating GNSS stations in the region, the eddy nature of modern geodynamic movements was established as an indicator of active tectonic disturbances. This allows, on the basis of modeling, to identify active geological structures, blocks, tectonic faults by analyzing the field of displacements and deformations, which is necessary to predict the locations of possible seismic events and substantiate preventive measures to ensure the safety of the people, industrial facilities, etc. [10].

Other issues of visualizing geodynamic movements upon the results of monitoring measurements that require further elaboration, but are not touched upon at this stage, are:

- Final implementation in the form of a computer software based on the developed mathematical apparatus for assessing the divergence of vector fields, enabling to identify the divergence based on the results of the initial geodetic data.

- Solving the issue of the possibility of increasing the promptness of obtaining the results of geodynamic studies through the use of exact ephemerides of different classes in the computations (Rapid vs Final). At the present time, two series of experiments have been 
carried out, which showed contradictory data, and a third series is being prepared for conducting.

- Solving the issue of choosing the minimum possible interval between the cyclic series of instrumental measurements, at which the errors of single measurements will have a minimum impact on the result of finding the velocities of modern geodynamic movements.

The paper was created within the framework of state assignment No. 075-00581-19-00 on topic No. 0328-2019-0005.

\section{References}

1. V.I. Utkin, A.A. Belousova, D.S. Tyagunov, D.V. Balandin, Reports of the Academy of Sciences, 431(2), 246-251 (2010)

2. A.A. Panzhin, Bulletin of the Nosov Magnitogorsk State Technical University, 1(49), 22 $26(2015)$

3. J. F. Zumberge, M. B. Heflin, D. C. Jefferson, M. M. Watkins et al., Journal of Geophysical Research: Solid Earth (1978-2012), 102(B3), 5005-5017 (1997)

4. B.T. Mazurov, A.A. Panzhin, A.A. Silaeva, Geodesy and Cartography, 3, 35-40 (2016) DOI: 10.22389 / 0016-7126-2016-909-3-25-40.

5. A.A. Panzhin, N.A. Panzhina, Mining Journal, 6, 31-40 (2019) DOI: 10.21440 / 05361028-2019-6-31-40.

6. B.T. Mazurov, Mathematical modeling in the study of geodynamics (Novosibirsk:

SGUGiT, 2019)

7. A.V. Vikulin, Processes in geomedia, 7, 194-206 (2016)

8. V.G. Kolmogorov, B.T. Mazurov, A.A. Panzhin, Geodesy and Cartography, 79(10), 4653 (2018) DOI: 10.22389 / 0016-7126-2018-940-10-46-53.

9. B.T. Mazurov, M.G. Mustafin, A.A. Panzhin, Bulletin of the Institute of Mining, 238(4), 376-382 (2019) DOI: 10.31897 / PMI.2019.4.376.

10. A.D. Sashurin, A.A. Panzhin, Mining information and Analytical Bulletin, 3-1, 188-198 (2020) DOI: 10.25018 / 0236-1493-2020-31-0-188-198. 